\title{
Influence of quasi-bound states on the carrier capture into quantum dots
}

Magnúsdóttir, Ingibjörg; Uskov, A.; Bischoff, Svend; Tromborg, Bjarne; Mørk, Jesper; Ferreira, R.; Bastard, G.

Published in:

Summaries of Papers Presented at the Quantum Electronics and Laser Science Conference, 2002. QELS '02.

Technical Digest.

Link to article, DOI:

10.1109/QELS.2002.1031281

Publication date:

2002

Document Version

Publisher's PDF, also known as Version of record

Link back to DTU Orbit

Citation (APA):

Magnúsdóttir, I., Uskov, A., Bischoff, S., Tromborg, B., Mørk, J., Ferreira, R., \& Bastard, G. (2002). Influence of quasi-bound states on the carrier capture into quantum dots. In Summaries of Papers Presented at the Quantum Electronics and Laser Science Conference, 2002. QELS '02. Technical Digest. IEEE.

https://doi.org/10.1109/QELS.2002.1031281

\section{General rights}

Copyright and moral rights for the publications made accessible in the public portal are retained by the authors and/or other copyright owners and it is a condition of accessing publications that users recognise and abide by the legal requirements associated with these rights.

- Users may download and print one copy of any publication from the public portal for the purpose of private study or research.

- You may not further distribute the material or use it for any profit-making activity or commercial gain

- You may freely distribute the URL identifying the publication in the public portal 
2. J. Urayama, et al., "Observation of phonon bottleneck in quantum dot electronic relaxation," Phys. Rev. Lett. 86, 4930-4933 (2001).

3. J. Bloch, et al., "Optical properties of multiple layers of self-organized InAs quantum dots emitting at $1.3 \mu \mathrm{m}$," App. Phys. Lett. 77, 2545-2547 (2000).

4. T.S. Sosnowsky, et al., "Rapid carrier relaxation in $\mathrm{In}_{0.4} \mathrm{Ga}_{0.6} \mathrm{As} / \mathrm{GaAs}$ quantum dots characterized by differential transmission spectroscopy," Phys. Rev. B 57, R9423-R9426 (1998).

\section{QThB4}

8:45 am

Electron-phonon Scattering In Metal
Clusters: Size Effects

N. Del Fatti, P. Langot and F. Vallée, CPMOH, CNRS--Université Bordeaux I, 351 cours de la Libération, 33405 Talence, France, Email: fvallee@cpmoh.u-bordeaux.fr

Ultrafast interactions of electrons between themselves and with their environment (lattice, surfaces ... .) are key effects in the fundamental and technological properties of metallic systems. Their modification by the confinement in metal nanoparticles is thus of central-interest for the understanding of the specific properties of these materials. Time resolved femtosecond techniques have been shown to be powerful tools for the investigation of the electron scattering processes in metals and have been extensively used in bulk materials ${ }^{1}$ and recently applied to metal nanoparticles. $^{2-4}$

However, the impact of confinement on electron-lattice energy exchanges has been addressed essentially in the strong excitation regime (i.e., for a large heating of the electrons). A large influence of the surrounding matrix has been demonstrated, ${ }^{5}$ precluding measurement of the intrinsic electron-phonon scattering in nanoparticles. In particular, contradictory results on the size effect have been reported (for a discussion see [4]). We have investigated the electron-lattice energy ex-

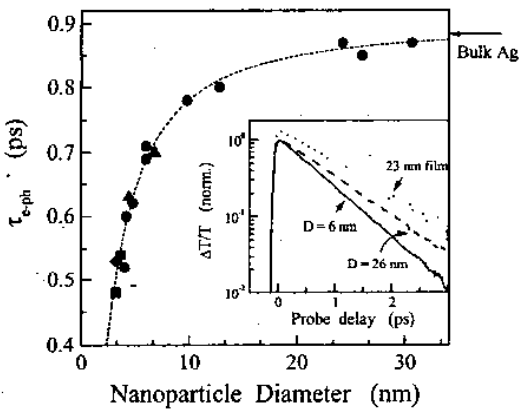

QThB4 Fig. 1. Measured electron-lattice energy exchange time $\tau_{\text {e-ph }}$ in silver nanoparticles embedded in a glass (circles), $\mathrm{Al}_{2} \mathrm{O}_{3}$ (squares) or $\mathrm{MgF}_{2}$ (diamond) matrix or in solution (triangles) as a function of their diameter. The inset shows the time dependence of the measured differential transmission $\triangle T / T$ on a logarithmic scale in $D=$ $.6 \mathrm{~nm}$ and $26 \mathrm{~nm} \mathrm{Ag}$ nanoparticle samples and in a Ag film (upshifted for clarity). changes in silver and gold nanoparticles in the weak excitation regime (induced electron temperature rise $\Delta T_{e}$ smaller than $100 \mathrm{~K}$ ). The results were found to be independent of the nanoparticle environment permitting to analyze the effect of particle size reduction on the intrinsic electronlattice coupling.

Experiments were performed in spherical silver and gold nanoparticles embedded either in a glass, $\mathrm{Al}_{2} \mathrm{O}_{3}$ or $\mathrm{MgF}_{2}$ matrix or in solution. Their average diameter $D$ ranged from 30 to $3 \mathrm{~nm}$. An athermal electron distribution was first created by absorption of a $25 \mathrm{fs}$ pulse delivered by a femtosecond Ti:sapphire oscillator. The induced changes of the sample transmission, $\Delta T / T$ was probed using a second pulse, with a frequency well below the interband absorption threshold (about $4 \mathrm{eV}$ in $\mathrm{Ag}$ and $2.3 \mathrm{eV}$ in $\mathrm{Au}$ ), to avoid spurious effects due to electron gas thermalization. ${ }^{3}$ For these probing conditions, the $\Delta T / T$ amplitude is proportional to the electron gas excess energy and its decay reflects electron energy losses to the lattice. Because of the high sensitivity of our appartus, we have been able to perform measurements for weak electron excitation $\left(\Delta T_{e}\right.$ in the $10-100 \mathrm{~K}$ range).

Measurements performed in silver and gold nanoparticles show an exponential decay of $\Delta T / T$ (Fig. 1), in agreement with the two-temperature model in the weak perturbation regime. ${ }^{1}$ The decay time has been found to be independent of the energy injected in the electron gas and of the matrix and sample preparation technique, showing that the electron-lattice energy exchange time $\tau_{\text {e-ph }}$ intrinsic to the nanoparticles is determined. $\tau_{\mathrm{e}-\mathrm{ph}}$ is almost identical to the one measured in the bulk materials for large nanoparticles $(D \geq 10$ $\mathrm{nm}$ ) but strongly decreases for smaller ones (Fig. 1), with similar behavior in gold and silver nanoparticles. This acceleration of the energy exchanges demonstrate confinement induced increase of the intrinsic electron-lattice interactions.

The measured $\tau_{e-p h}$ size dependence is comparable to the one recently reported for the electron-electron scattering time and will be discussed using the same approach, i.e., introducing reduction of the electron-ion interactions due spatial electron distribution change close to the nanoparticle surface. ${ }^{3}$

\section{References}

1. C.K. Sun, F. Vallée, L. Acioli, E.P. Ippen and J.G. Fujimoto, "Femtosecond tunable measurement of electron thermalization in gold", Phys. Rev. B 50, 15337 (1994).

2. T. Tokizaki, A. Nakamura, S. Kavelo, K. Uchida, S. Omi, H. Tanji, and Y. Asahara, "Subpicosecond time response of thirdorder nonlinearity of small copper particles in glass", Appl. Phys. Lett. 65, 941 (1994)

3. C. Voisin, D. Christofilos, N. Del Fatti, F Vallee, B. Prével, E. Cottancin, J. Lermé, M. Pellarin, and M. Broyer "Size dependent electron-electron interactions in metal nanoparticles", Phys. Rev. Lett. 85, 2200 (2000).

4. C. Voisin, N. Del Fatti, D. Christofilos, and F. Vallée, "Ultrafast electron dynamics and optical nonlinearities in metal nanoparticles" $\mathrm{J}$. Phys. Chem B 105, 2264 (2001).

5. Y. Hamanaka, J. Kuwabata, I. Tanahashi, S Omi, and A. Nakamura, Phys. Rev. B 63 (2001).
Influence of Quasi-bound States on the Carrler Capture into Quantum Dots

I. Magnusdottir, A. Uskov, S. Bischoff, B. Tromborg, and J. Mork, COM Education \& Research Center, Technical University of Denmark, Bldg. 345 w, DK-2800 Kgs. Lyngby, Denmark,Email: in@com.dtu.bk

R. Ferreira and G. Bastard, Laboratoire de Physique ENS, 24 rue Lhomond, F-75005 Paris, France

An important characteristic of quantum dot (QD) materials is the timescale on which carriers are captured into the dots and relax to their ground state. The properties of devices based on QDs, such as lasers, thus rely on efficient carrier feeding to the active QD states. These processes are believed to be mediated by carrier-phonon and carrier-carrier interaction (Auger processes). ${ }^{1,2}$ In systems of higher dimensionality, carrier relaxation via emission of LO (Longitudinal Optical) phonons is dominant. However, due to the discrete QD density of states, this process is often considered impossible unless the energy level separation equals the LO phonon energy, leading to a so-called phonon bottleneck. ${ }^{3}$ This argument is based on the assumption that the carrier-LO phonon interaction is weak.

It was shown ${ }^{4,5}$ that carriers in discrete QD states couple strongly to phonons and that the intersubband transition cannot be treated with Fermi's golden rule. Here, we extend the analysis to the coupling between carriers in quasi-bound continuum states and discrete QD states. In previous calculations of carrier capture rates into $\mathrm{QDs},{ }^{1,2}$ the incident carrier wavefunctions have been considered to be plane waves, neglecting the influence of the QD quasi-bound states. We will demonstrate that these states couple strongly to the phonon bath. We consider a spherical dot of radius $a$, with a finite confinement potential, $V_{0}$, in the effective mass approximation, where the states are characterized by the angular momentum quantum numbers $(h m)$. We focus our attention on QDs with one discrete state $(h=0) . \mathrm{V}_{0}$ and $a$ are chosen such that a quasi-bound state $\left(l_{v}=1\right)$ and the discrete state are separated by $\hbar \omega_{10}=35 \mathrm{meV}$. The quasi-bound state is traced by the probability that the carrier is present within the QD volume, shown in Fig. 1(a). Fig. l(b) shows the probability when the carrier-LO phonon interaction has been taken into account. In this case, two polaron states, labelled $|1\rangle$ and $|2\rangle$, appear. As an important and surprising result, we find that $|1\rangle$ corresponds to a discrete state confined to the QD. These polaron states would be stable if both the electrons and phonons were stable. It is known from bulk structures that the LO phonon lifetime is $1 / \Gamma_{\mathrm{ph}} \approx 2 \mathrm{ps}$ at room temperature. ${ }^{6}$ The polaron thus decays due to the decay of its.phonon part. The relaxation rate of $|1\rangle$ to the $l=0$ state can be found by weighting $\Gamma_{\mathrm{ph}}$ by the phonon part of the total probability. We calculate the relaxation times to be close to 4 ps. Fig. 2 shows the case for holes, where the resonance is broader. Nevertheless, a discrete polaron state is found within the dot. In this case, we also calculate a capture time of the order $4 \mathrm{ps}$.

It should be noted that in the situation of more confined QDs with more than one discrete level and a quasi-bound state situated $\hbar \omega_{\omega}$ above 


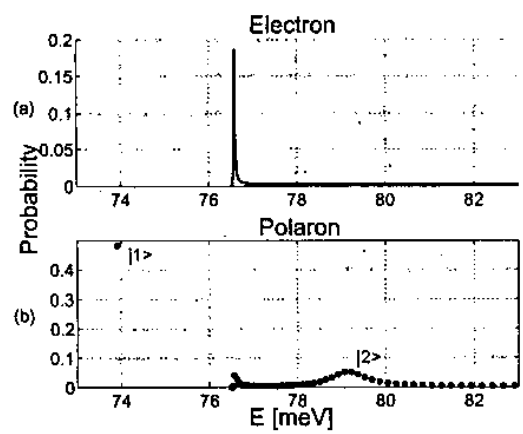

QThB5 Fig. 1. (a) The probability that an electron state with $l_{v}=1$ in the continuous part of the energy spectrum is present within the volume of the $\mathrm{QD}$, neglecting the carrier-LO phonon interaction. A quasi-bound state is situated at $E_{1}=$ $76.58 \mathrm{meV}$ with a FWHM of $0.025 \mathrm{meV}$. The discrete $l=0$ state (not shown) is situated at $E=$ $41.59 \mathrm{meV}, \hbar \omega_{\omega}$ below the quasi-bound state (b) Taking carrier-LO phonon interaction into account, two polaron states $(|1\rangle, 2\rangle)$ appear. One, $|1\rangle$, is discrete and situated within the dot (at $E_{0}$ $=73.9 \mathrm{meV}) .|2\rangle$ is a broader level centered at $E$ $=79.1 \mathrm{meV}$ and the level splitting is $5.2 \mathrm{meV}$. In between them, a reminescence of the quasibound state is seen. These results are independent of the quantum number $m_{v}$. We use $a=8.55 \mathrm{~nm}$, the carrier mass $\mathrm{m}^{*}=0.067 \mathrm{~m}_{0}$ and $\mathrm{V}_{0}=76.49$ meV.

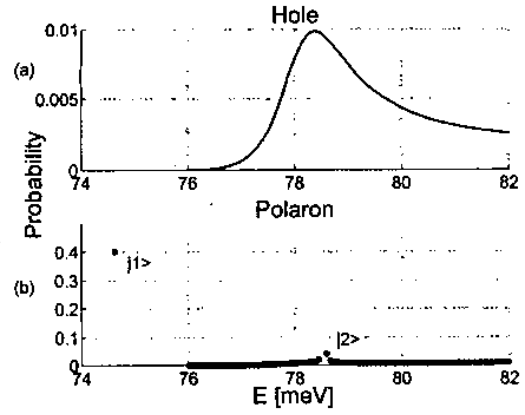

QThB5 Fig. 2. As Fig. 1 but for holes. The quasi-bound state seen in (a) when carrier-LO phonon interaction is neglected is broad compared to the case for electrons. It is situated at $E_{1}$ $=78.34 \mathrm{meV}$ with a FWHM of $2.1 \mathrm{meV}$. The discrete $l=0$ state (not shown) is situated at $E_{0}=$ $43.5 \mathrm{meV}, \hbar \omega_{\mathrm{LO}}$ below the quasi-bound state. However, the polaron states appearing when the interaction is taken into account (b) still contain a discrete level, $[1\rangle$, confined to the $\mathrm{QD}$, at $E=$ $74.6 \mathrm{meV}$. The state $|2\rangle$ is centered at $E=78.7$ $\mathrm{meV}$, so the level splitting is $4.1 \mathrm{meV}$. The $\mathrm{QD}$ radius is $a=3.662 \mathrm{~nm}$, the carrier mass $\mathrm{m}^{*}=0.34$ $\mathrm{m}_{0}$ and $\mathrm{V}_{0}=76.0 \mathrm{meV}$.

a discrete state, similar results are expected. In conclusion, we have shown that QD quasi-bound states couple strongly to phonons and that the corresponding polaron states may mediate efficient carrier capture to the QD ground state.
References

1. A.V. Uskov, J. McInerney, et al., "Auger carrier capture kinetics in self-assembled quantum dot structures," Appl. Phys. Lett., 72, 58-60 (1998).

2. R. Ferreira and G. Bastard, "Phonon-assisted capture and intradot Auger relaxation in quantum dots," Appl. Phys. Lett., 74, 2818-2820 (1999).

3. H. Benisty, C.M. Sotomayor-Torres, and C. Weisbuch, "Intrinsic mechanism for the poor luminescence properties of quantum-box systems," Phys. Rev. B, 44, 10945-10948 (1991).

4. T. Inoshita and H. Sakaki, "Density of states and phonon-induced relaxation of electrons in semiconductor quantum dots," Phys. Rev. $B, 56, \mathrm{R} 4355-\mathrm{R} 4358$ (1997).

5. O. Verzelen, R. Ferreira, and G. Bastard, "Polaron lifetime and energy relaxation in semiconductor quantum dots," Phys. Rev. B, 62, R4809-R4812 (2000)

6. F. Vallee and F. Bogani, "Coherent time-resolved investigation of $\mathrm{LO}$-phonon dynamics in GaAs," Phys. Rev. B, 43, 12049-12052 (1991)

\section{QThB6}

9:15 am

Dipole-forbidden Energy Transfer between CuCI Quantum Cubes via Optical Near-field

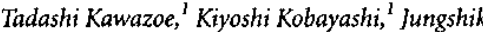
Lim, ${ }^{2}$ Yoshihito Narita, ${ }^{3}$ and Motoichi Ohtsu, ${ }^{1,2}$ ${ }^{\prime}$ Exploratory Research for Advanced Technology (ERATO), Japan Science and Technology Corporation (JST), 687-1 Tsuruma, Machida, Tokyo 194-0004, Japan, Email. kawazoe@ohtsu.jst.go.jp; ${ }^{2}$ Interdisciplinary Graduate School of Science and Engineering, Tokyo Institute of Technology, 4259 Nagatsuta, Midori-ku, Yokohama 226-8502, Japan; ${ }^{3}$ IASCO Corporation, 2967-5, Ishikawa-cho, Hachioji, Tokyo, 192-8537, Japan

The coupled quantum-dots system exhibits more unique properties in contrast with the single quantum-dot system. ${ }^{1}$ The optical near-field interaction ${ }^{2}$ is interesting, as it can govern the coupling strength among quantum dots. Recently, an ultra-fast energy transfer between bacteriochlorophyll molecules via the optical near field was theoretically shown. ${ }^{3}$ This kind of the energy transfer is possible for the neighboring quantum dots system.

The quantized exciton energy levels of $(1,1,1)$ in a $\mathrm{CuCl}$ quantum cube $(\mathrm{QC}) \mathrm{X}(4.6 \mathrm{~nm})$ and $(2,1,1)$ in a QC Y $(6.3 \mathrm{~nm})$ resonate with each other. ${ }^{4}$ For this type of resonant condition, the energy transfer time between QC via the optical near-field is estimated at $40 \mathrm{ps}$, assuming that the separation between two QCs is equal to $10 \mathrm{~nm}^{5}$ This energy transfer time is much shorter than the exciton lifetime and is longer than the intersub-level transition $\tau_{\text {sub }}$. Therefore, most of the exciton in a $4.6-\mathrm{nm} \mathrm{CuCl} \mathrm{QC} \mathrm{transfer} \mathrm{to} \mathrm{the}$ neighboring 6.3-nm QC. Here, we note that this energy transfer is optically forbidden, but becomes allowed with the optical near field.

Figure 1(b) shows a far-field luminescence spectrum at $15 \mathrm{~K}$. It represents the luminescence intensity from large numbers of $\mathrm{CuClQCs}$, and is

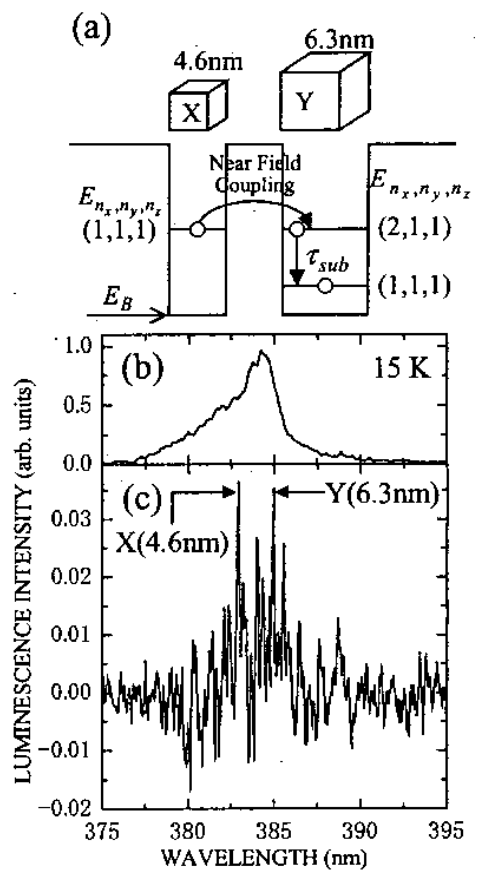

QThB6 Fig. 1. (a): Schematic drawings of closely located $\mathrm{CuCl}$ quantum cubes $\mathrm{X}$ and $\mathrm{Y}$ with the effective side lengths of $4.6 \mathrm{~nm}$ and $6.3 \mathrm{~nm}$, respectively. Their exciton energy levels $n_{x}, n_{y}$, and $n_{z}$ represent quantum numbers of an exciton. (a) and (b): Far-field and near-field luminescence spectra of $\mathrm{CuCl} \mathrm{QCs}$ embedded in $\mathrm{NaCl}$ matrix at $15 \mathrm{~K}$.

inhomogeneously broadened owing to the size distribution of the QCs. Figure l(c) represents a near-field luminescence spectrum with many fine structures. The structures are due to the contribution of the QCs near the apex of the near-field probe with a 50-nm aperture. The peaks $\mathrm{X}$ and $\mathrm{Y}$ correspond to the confined $Z_{3}$-exciton energy levels of the QCs with side lengths of $4.6 \mathrm{~nm}$ and $6.3 \mathrm{~nm}$, respectively.

Figures 2(a) and (b) show the near-field optical microscope images for peaks $X$ and $Y$ in Fig. 1 (c), respectively. These images clearly establish anti-correlation features in their intensity distributions, as manifested by the dark and bright regions surrounded by broken curves.

Figure 2(c) shows the values of the cross-correlation coefficient $C$ between the spatial distributions of the luminescence intensity from a 6.3$\mathrm{nm} \mathrm{QC}$ and that from a different size QCs. The large negative value of $C$ identified by an arrow $(2,1,1)$ clearly shows the anti-correlation feature between Figs. 2(a) and (b). This anti-correlation feature can be understood by the energy transfer from $X$ cubes to $Y$ cubes. A 4.6-nm QC-, 'accidentally' located close to a $6.3-\mathrm{nm}$ QC cannot emit light, but instead transfers the energy to the 6.3$\mathrm{nm} \mathrm{QC}$. As a result, in the region containing embedded $6.3-\mathrm{nm} \mathrm{QCs}$, the luminescence intensity from 4.6-nm QCs is low while it from 6.3-nm QCs is high. As explained in Fig. 1 , it is reasonable to attribute the origin of the interaction to the near-field energy transfer.

For $\mathrm{CuCl}$ quantum cubes in a $\mathrm{NaCl}$ matrix other possibility of energy transfer, such as carrier 